\title{
EDITORIAL
}

\section{Utilidad práctica de la monitorización de la frecuencia cardiaca durante el ejercicio físico}

\section{Practical usefulness of heart rate monitoring in physical exercise}

\author{
Camilo E. Povea ${ }^{\mathrm{a}, \mathrm{b}, *}$ y Arturo Cabrera ${ }^{\mathrm{c}}$ \\ a Servicio Unidad de prevención cardiovascular y medicina del deporte, Fundación Cardioinfantil-Instituto de Cardiología, \\ Bogotá, Colombia \\ b Departamento de Medicina interna, Postgrado Medicina del Deporte, Universidad Nacional de Colombia, Bogotá, Colombia \\ ' Escuela Nacional del Deporte, Cali, Colombia
}

Recibido el 24 de mayo de 2018; aceptado el 25 de mayo de 2018

Disponible en Internet el 1 de junio de 2018

La práctica de actividad física dirigida y programada, o incluso la promoción de un estilo de vida más activo, son cada vez más difundidas. De hecho, es claro que los beneficios de realizar estas actividades de forma regular constituyen un factor protector frente al desarrollo de patologías crónicas ${ }^{1}$. Estos efectos favorables se pueden evidenciar en prevención primaria y secundaria en pacientes con insuficiencia cardiaca, coronariopatía o hipertensión $\operatorname{arterial}^{2}$ y de manera más amplia para cualquier individuo sedentario con o sin factores de riesgo cardiovascular.

De acuerdo con este panorama, la cantidad de personas que se pueden beneficiar de un programa de actividad física personalizada es cada vez mayor, fenómeno que hace que la utilización de métodos que permitan agilizar y facilitar la realización de dichos programas, sea más frecuente. Para los profesionales de la salud y para el médico en particular, es indispensable dar recomendaciones sobre la mejor forma de poder cuantificar la intensidad de un esfuerzo físico determinado, así como de la utilización correcta y la determinación de la frecuencia cardiaca (frecuencia cardiaca) como indicador de esta intensidad de esfuerzo. La determinación de

\footnotetext{
* Autor para correspondencia.

Correo electrónico: cpovea@cardioinfantil.org (C.E. Povea).
}

la frecuencia cardiaca mediante el método manual, puede arrojar resultados imprecisos ${ }^{3}$ e incluso, en algunos casos, requerir de la interrupción del esfuerzo para su correcta utilización, alterando el resultado real. El uso del electrocardiograma y/o el Holter resulta imposible de manera regular y su bondad en el terreno es prácticamente nula.

La metodología de monitorización del entrenamiento en el campo de las actividades físicas o deportivas (calificadas como de resistencia) ha progresado de manera importante ${ }^{4}$. De la utilización casi exclusiva de la percepción subjetiva de esfuerzo, se ha logrado un estado de planificación del entrenamiento mucho más individualizado, ajustado, racionalizado y en cierta medida mucho más eficaz. La utilización de dispositivos que permite medir simultáneamente múltiples variables tanto internas (frecuencia cardiaca, frecuencia respiratoria, temperatura) como externas (acerelometría, velocidad, altura, temperatura, etc. $)^{5}$, ha permitido supervisar de manera específica la intensidad e incluso la duración de la actividad física ${ }^{4,6}$.

El seguimiento científico de las sesiones de entrenamiento ha significado un avance considerable en el control fisiológico de la adaptación del ser humano al esfuerzo físico. La masificación de la utilización de dispositivos y aplicaciones que permiten monitorizar constantemente la frecuencia cardiaca durante el ejercicio o hasta en 
competición ${ }^{7}$, facilita la inclusión de las personas en eventos deportivos para el público en general (carreras de $10 \mathrm{Km}$, ciclo-turismo, caminatas, etc.) En el campo de las ciencias aplicadas al deporte y la medicina del deporte aplicada a la clínica, la aparición de numerosos y modernos dispositivos de monitorización de los individuos es cada vez más corriente. De manera general puede decirse que existen grandes grupos de sensores: sensores de movimiento (podómetros, acelerómetros y GPS) y otros de tipo fisiológico (cardiofrecuenciómetros, sensores de temperatura y sensores integrados $)^{5}$, que contribuyen a mejorar la confianza de quien realiza el ejercicio y otorgan cierto margen de seguridad en el plano cardiovascular. Sin embargo, en ocasiones durante el esfuerzo físico, las personas pueden referir cifras de frecuencia cardiaca que suelen ser anormalmente elevadas; por consiguiente es importante recordar que aunque los cardiofrecuenciómetros fueron diseñados para atletas sanos con ritmo sinusal de base, estos aparatos son capaces de detectar arritmias inducidas por el ejercicio, pero no permiten detectar la morfología de los complejos QRS ni mucho menos, las señales auriculares ${ }^{8}$, de ahí que su uso deber ser cuidadoso. Pese a que numerosos profesionales de la salud pueden llegar a reconocer de manera amplia el interés de monitorizar la frecuencia cardiaca durante el esfuerzo de manera permanente, con mucha frecuencia los monitores del ritmo cardiaco siguen siendo mal utilizados o abandonados por los usuarios y pacientes una vez transcurrido un tiempo, cuando sus funciones, aun las más básicas, resultan incomprendidas.

\section{La frecuencia cardiaca como indicador del nivel de esfuerzo del organismo}

Tratándose de ejercicio físico, la relación entre potencia muscular o intensidad de ejercicio en carrera es, de manera general, lineal. Para cualquier incremento de la potencia y/o intensidad corresponde un incremento de la frecuencia cardiaca; sin embargo, estos aumentos también pueden estar relacionados con el estrés asociado a la actividad y no necesariamente con el incremento de la carga de trabajo, característica que puede observarse más fácilmente en zonas en las cuales el ejercicio es poco intenso (cercano al reposo), en donde se pueden encontrar incrementos de hasta 40 latidos por minuto, sin que impliquen aumento de las demandas energéticas.

La presencia de ejercicio físico intenso e incremento de la carga de trabajo en ausencia de ascenso de la frecuencia cardiaca, se considera una meseta, la cual en muchos casos, se define como la frecuencia cardiaca máxima.

\section{Frecuencia cardiaca máxima}

La frecuencia cardiaca máxima $\left(\mathrm{FC}_{\max }\right)$ que cada individuo alcanza tiene un carácter totalmente individual y está bajo la influencia del comando nervioso central (pero también existen condiciones ambientales que pueden influenciarla (temperatura, higrometría, altura, etc. $)^{10}$. De esta manera, la relación entre la frecuencia cardiaca y el consumo de oxígeno es un dato puramente individual ${ }^{10,11}$. La frecuencia cardiaca máxima disminuye con la edad, posiblemente debido a modificaciones en la respuesta autonómica, cambio en la sensibilidad de los cardiomiocitos a las catecolaminas y/o reducción de la recaptación de las mismas y cambio en la sensibilidad del barorreflejo ${ }^{14}$.

La utilización indiscriminada de la ecuación 220 - edad para calcular la $\mathrm{FC}_{\max }$, sobreestima la cifra de frecuencia cardiaca en jóvenes y subestima la de los individuos mayores $^{12}$; una investigación longitudinal demuestra un menor descenso en la $F C_{\max }$ con la edad $(0,7$ en lugar de 1 latido por año) y un punto de intersección-y más bajo sin importar el nivel de entrenamiento o el género ${ }^{11}$. Aparentemente el valor de $\mathrm{FC}_{\text {max }}$ puede variar en función de la modalidad deportiva que se practique $^{10} \mathrm{o}$ del nivel de entrenamiento; para esfuerzos equivalentes en potencia (carrera vs. esfuerzo en bicicleta) para un mismo individuo en condiciones ambientales similares, la frecuencia cardiaca alcanzada durante el atletismo puede ser alrededor de 10 latidos por minuto, más elevada que en el ciclismo ${ }^{10}$. De hecho se sugieren fórmulas diferentes para calcular el esfuerzo máximo según el deporte [165 - 0,5 x edad (años) \pm 5 latidos, para atletismo; $160-0,5 \times$ edad (años) \pm 5 latidos para ciclismo]. Si se consideran deportes en los cuales la posición corporal puede modificar el retorno venoso, tales como la natación, se pueden apreciar frecuencias cardiacas máximas inferiores en 5 a 10 latidos $^{13}$.

\section{Deriva cardiovascular}

Durante un esfuerzo de larga duración, en un ejercicio de más de 20 minutos, con una intensidad constante, se presenta una deriva de la frecuencia cardiaca, que equivale a un incremento de la frecuencia cardiaca9. Esta deriva es ocasionada por un descenso progresivo del volumen de eyección sistólica relacionado con la deshidratación del sujeto, particularmente con temperaturas ambientales elevadas que conllevan disminución de la volemia eficaz $(100-120 \mathrm{ml})$, lo cual debe ser compensado por una aceleración de la frecuencia cardiaca para garantizar el mismo aporte de oxígeno a los músculos implicados. De esta forma, si en la práctica se aprecia un aumento de la frecuencia cardiaca durante la sesión de entrenamiento pese a mantener una intensidad constante, se debe promover la rehidratación para evitar la deriva cardiovascular.

\section{Zonas de frecuencia cardiaca}

La mayoría de los programas informáticos o aplicaciones que acompañan actualmente a los dispositivos de frecuencia cardiaca y que son utilizados para el entrenamiento personalizado reconocen y utilizan cinco zonas de frecuencia cardiaca, clasificadas de la 1 a $5^{9}$. El método válido de determinación de zonas de entrenamiento con frecuencia cardiaca es una prueba de umbral de lactato en el laboratorio en una banda sinfín o en un cicloergómetro, que implique un aumento de la carga de trabajo por etapas y la obtención de muestras de sangre al final de cada etapa. Estas zonas pueden ser intervalos de frecuencia cardiaca de 5 a 10 latidos por minuto de cada individuo; la determinación también puede realizarse mediante una ergoespirometría que se hace necesaria tanto en sujetos con pobre capacidad física por procesos patológicos pulmonares o cardiovasculares, como en los deportistas de alto rendimiento. De esta 
manera se establece el pico máximo y el primer umbral aeróbico o de resistencia (adaptación ventilatoria), y el segundo umbral anaeróbico o de resistencia. Sin embargo, la interpretación de los umbrales, en muchos casos es dependiente del operador.

La frecuencia cardiaca máxima prevista para la edad es un valor que, en general, no corresponde a la realidad. La mejor manera de establecerla es a través de un test de laboratorio o de terreno adaptado para el sujeto que lo requiera; éste puede ser útil para sujetos sanos asintomáticos y sin factores de riesgo ni antecedentes familiares.

\section{Frecuencia cardiaca de reposo}

Su determinación inicial para realizar los cálculos, no es absolutamente indispensable y pueden existir muchos factores que la condicionan y variar de un día a otro. Para su determinación correcta se recomienda realizarla en la mañana, antes de levantarse o inmediatamente después del despertar, preferiblemente en decúbito supino, en ayunas, al menos cinco minutos durante tres días consecutivos y repetirla con frecuencia. Si bien esta puede ser un buen indicador de condición física o en casos de alteración pueden revelar un estado de sobrecarga física o emocional, no permite predecir con certeza qué tanto cambia con el esfuerzo físico.

\section{Frecuencia cardiaca de recuperación}

El seguimiento de esta variable puede resultar muy interesante, siempre y cuando se tenga precisión a la hora de establecer el esfuerzo al cual se ha sometido el individuo. Adicionalmente, la frecuencia cardiaca de recuperación depende del nivel de forma y es poco sensible a factores externos ${ }^{17}$.

En términos prácticos se considera que un sujeto tiene buena capacidad de recuperación después del esfuerzo, si su frecuencia cardiaca disminuye al menos una tercera parte con relación a la máxima alcanzada durante el ejercicio al tercer minuto de recuperación pasiva ${ }^{18}$.

\section{Frecuencia cardiaca de reserva}

Se utiliza para establecer las zonas y corresponde a la diferencia entre la frecuencia cardiaca máxima y la frecuencia cardiaca de reposo. Es evidente que cuanto más elevada sea, más relevancia cobra como indicador de esfuerzo y será más importante para determinar el papel de la frecuencia cardiaca en la progresión del consumo de oxígeno $\left(\dot{\mathrm{V}} \mathrm{O}_{2}\right)$ al esfuerzo.

\section{Zonas de trabajo}

El método de Karvonen establece una frecuencia objetivo, que es igual a la frecuencia cardiaca de reposo más un porcentaje de la frecuencia cardiaca de reserva ${ }^{15}$. Frecuencia cardiaca reserva = (frecuencia cardiaca reposo - frecuencia cardiaca máxima), para calcular la frecuencia cardiaca de entrenamiento se toma el $85 \%$ de la frecuencia cardiaca reserva previamente calculada y se le adiciona la frecuencia cardiaca de reposo. Si bien este método resulta muy preciso, está condicionado a las variaciones de la frecuencia cardiaca de reposo descritas. El método más simple es aquel que clásicamente utiliza el porcentaje (\%) de la frecuencia cardiaca máxima y el porcentaje de $\mathrm{VO}_{2}$ que se mantiene casi idéntico en cualquier individuo que realiza una sesión al $85 \%$ de su $\dot{V} \mathrm{O}_{2 \text { max }}$ que corresponde más o menos a solicitar entre el 90-95\% de su frecuencia cardiaca máxima.

\section{Zonas de entrenamiento}

Zona 1. Los valores de frecuencia cardiaca se emplean en especial para los procesos de recuperación y para trabajar la resistencia a la fatiga y adicionalmente la eficiencia. Los valores corresponden al rango de 50 a $60 \%$ de la frecuencia cardiaca máxima.

Zona 2. Para el entrenamiento de resistencia. La intensidad de esta zona produce mejorías cardiovasculares con el entrenamiento, pero no se requiere de más tiempo. Los valores corresponden al rango de 60 a $70 \%$ de la frecuencia cardiaca máxima.

Zona 3. Es de mayor intensidad, y se utiliza para el entrenamiento de resistencia mayor. Esta es la zona de uso común para el entrenamiento de tiempos. El volumen de entrenamiento de la zona 3 debe ser limitado (especialmente en carrera), debido a la presión impuesta sobre el organismo con esta intensidad del entrenamiento. Los valores corresponden al rango de 70 a $80 \%$ de la frecuencia cardiaca máxima.

Zona 4. La intensidad es muy cercana al umbral de lactato de un atleta. Los trabajos en esta zona son considerados intensos, tanto física como psicológicamente y se deben utilizar generalmente al menos una vez por semana durante un entrenamiento planificado. Sin embargo, debe ser vigilado y limitado, puesto que en ella la probabilidad de sufrir lesiones osteomusculares puede verse incrementada. Los valores corresponden al rango de 80 a $90 \%$ de la frecuencia cardiaca máxima.

Zona 5. Se utiliza para entrenar el $\dot{V} O_{2 \max }$ y debe hacerse con moderación, debido a la sobrecarga extrema a la cual se puede conducir. $\mathrm{El} \dot{V} \mathrm{O}_{2 \max }$ está determinado genéticamente y una vez que se alcanza el límite máximo de éste, no se puede aumentar de manera significativa. El tiempo de entrenamiento debe ser empleado en otros lugares. Los valores corresponden al rango de 90 a 100\% de la frecuencia cardiaca máxima.

Otra aproximación para la monitorización del entrenamiento es la cuantificación de la carga de entrenamiento, la cual considera dos conceptos fundamentales; por un lado la carga interna, que representa el estrés fisiológico al esfuerzo realizado durante las sesiones de entrenamiento, y por otro lado, la carga externa correspondiente a las variables externas del entrenamiento, como duración, velocidad, aceleración, vatios, etc. ${ }^{20}$

Para el cálculo de la carga de entrenamiento se puede usar la siguiente fórmula:

Carga del entrenamiento = carga interna $\mathrm{x}$ carga externa (medida en unidades arbitrarias)

Dentro de las variables fácilmente cuantificables de la carga interna, se encuentran la frecuencia cardiaca, mien- 
tras que para la carga externa, el tiempo de duración de la sesión resulta ser una forma práctica de medición ${ }^{20}$.

Tradicionalmente la monitorización del entrenamiento se centra solo en una de las dos variables que componen la carga, lo que resulta en una visión parcial del binomio estímulo-respuesta; de modo que integrar en la monitorización tanto la carga interna como la carga externa, puede facilitar la toma de decisiones en cuanto a la solicitud de trabajo para una sesión determinada, permitiendo la monitorización individual del entrenamiento.

Una manera de individualizar el entrenamiento por medio de la monitorización de la carga, es el método TRIMP (training impulse), que corresponde a un modelo matemático, que permite cuantificar la carga total de una sesión de ejercicio ${ }^{20}$ integrando la carga interna (frecuencia cardiaca) con la carga externa (tiempo de sesión).

Para su cálculo es necesario conocer la frecuencia cardiaca en reposo, la frecuencia cardiaca máxima (esta última medida en un esfuerzo máximo), la frecuencia cardiaca promedio de la sesión de ejercicio y el tiempo de dicha sesión ${ }^{21}$.

TRIMP $=\mathrm{D} \times(\Delta \mathrm{FC})] \times \mathrm{e}^{(\mathrm{b} \times \Delta \mathrm{Fc})}$

Donde el $\triangle F C$ es igual a:

[(FC promedio en la sesión - FC reposo)/(FC máxima FC reposo)]

La constante $e=2,718$; el valor de $b$ refleja el grado de estrés del ejercicio, estando asociado con la curva de lactato durante el esfuerzo, que tiene un comportamiento diferente en hombres y mujeres, siendo para ellas $b=1,67$ y para ellos $b=1,92^{21}$.

Este modelo de cuantificación de la carga de entrenamiento es aplicable tanto a personas sanas $^{22}$ como para pacientes con enfermedad cardiaca ${ }^{23}$. En el caso de pacientes con falla cardiaca crónica, un programa de rehabilitación cardiaca hace parte de su tratamiento, con un nivel de evidencia $1^{24,25}$. Sin embargo, el óptimo estímulo en intensidad y duración es controvertido, ya que en recientes estudios muestra una tendencia a mayor beneficio con ejercicio en intervalos, al compararlo con ejercicio continuo ${ }^{26}$. Sin embargo, el estrés generado para cada método no necesariamente es el mismo, lo que dificulta la comparación entre ellos. lellamo et al. compararon dos programas de ejercicio por 12 semanas en pacientes con falla cardiaca, uno de carácter continuo y otro en intervalos. Utilizaron el método TRIMP para monitorizar de manera individual las cargas del entrenamiento, buscando que la carga total fuera la misma en ambos grupos. Encontraron un incremento del $\dot{V} \mathrm{O}_{2 \max }$ y el umbral ventilatorio en los dos métodos, sin diferencias significativas entre los grupos. Demostraron así que la monitorización individual de la carga de entrenamiento permite interpretar mejor el estímulo solicitado y sus posibles adaptaciones $^{23}$.

\section{Generalidades de los monitores de frecuencia cardiaca}

Los cardiofrecuencimetros son dispositivos electrónicos simples compuestos por dos elementos: el emisor (generalmente ubicado en el tórax) capta la señal eléctrica del corazón que equivale a la onda $\mathrm{R}$ del electrocardiograma ${ }^{3}$ y la envía hacia un reloj o dispositivo receptor que convierte en numéricos los datos, mostrando y almacenando los valo- res. Más recientemente se han incluido otros sistemas que detectan movimientos y velocidad de desplazamiento. La miniaturización de los dispositivos los ha tornado más sofisticados, de manera que ofrecen multiplicidad de funciones. Algunos modelos más recientes incluyen los emisores en la misma muñeca, aunque si se considera que un individuo puede realizar diferentes tipos de ejercicio, la precisión de estos últimos no supera a los que tienen sus electrodos de emisión en la región anterior del tórax. Estudios recientes muestran diversos grados de correlación con trazados electrocardiográficos dependiendo de la modalidad de ejercicio y/o la marca de los equipos, siendo el más adecuado de todos los emisores el que se ubica en el tórax, que sería el más recomendable en pacientes y deportistas ${ }^{19}$.

\section{Funciones básicas}

En la actualidad los avances obtenidos en el electrodo emisor no son equiparables a los de los receptores. Los materiales de los cuales están hechos los soportes sobre los que se instalan los electrodos, se han vuelto flexibles y por ende más cómodos; los captores muestran buenas correlaciones $(0,97)$ con los valores comparativos de referencia en pruebas de esfuerzo (3-4 latidos de diferencia) ${ }^{19}$. Actualmente, algunos emisores, que utilizan tecnología inalámbrica, se enlazan mediante aplicaciones a teléfonos inteligentes ${ }^{16}$. La mayor parte de modelos propuestos están codificados, lo cual permite evitar interferencias con otros dispositivos cercanos; así mismo, la selección de diferentes longitudes de onda hace difícil encontrar dos señales coincidentes. La frecuencia cardiaca en general se muestra en las pantallas cada 5 o cada 15 segundos en los modelos más básicos; solo algunos aparatos calificados como de gama alta proponen el almacenamiento latido a latido ${ }^{6}$; las zonas de trabajo se pueden memorizar y generalmente puede existir una 0 varias alarmas sonoras que indiquen los trabajos en ciertas zonas. Algunos dispositivos calculan el índice de forma física de manera subjetiva y lo equiparan al cálculo del consumo máximo de oxígeno. La precisión de este método es bastante discutible y su utilidad puede mejorarse siempre y cuando sea posible realizar medidas periódicas y en modelos que utilicen el registro de la frecuencia cardiaca latido a latido que favorezcan un análisis de la variabilidad del ritmo cardiaco $^{6,17}$.

En conclusión, el monitorización del entrenamiento debe hacerse de manera ordenada, sistemática y mediante herramientas prácticas que permitan conocer la carga de entrenamiento, teniendo, en la medida de lo posible, una monitorización tanto de la carga externa como la carga interna. Es en este último punto donde la frecuencia cardiaca desempeña un importante rol para la planificación y toma de decisiones de manera individual, tanto en personas sanas como en pacientes con enfermedad cardiaca.

\section{Bibliografía}

1. https://aspe.hhs.gov/basic-report/physical-activityfundamental-preventing-disease.

2. Lavie CJ, Thomas RJ, Squires RW, Allison TG, Milani RV. Exercise training and cardiac rehabilitation in primary and 
secondary prevention of coronary heart disease. Mayo Clin Proc. 2009;84:373-83.

3. Laukkanen RM, Virtanen PK. Heart rate monitors: state of the art. J Sports Sci. 1998;16 Suppl:S3-7.

4. Wright SP, Hall Brown TS, Collier SR, Sandberg K. How consumer physical activity monitors could transform human physiology research. Am J Physiol Regul Integr Comp Physiol. 2017; 312:R358-R367. First published January 4, 2017; doi:10.1152/ajpregu.00349.2016.

5. Li RT, Kling SR, Salata MJ, Cupp SA, Sheehan J, Voos JE. Wearable performance devices in sports medicine. Sports Health. 2016;8:74-8, http://dx.doi.org/10.1177/1941738115616917. Epub 2015.

6. Achten J, Jeukendrup AE. Heart rate monitoring: applications and limitations. Sports Med. 2003;33:517-38. PubMed doi:10.2165/00007256-200333070-00004.

7. Cardinale M, Varley MC. Wearable training-monitoring technology: applications, challenges, and opportunities. Int J Sports Physiol Perform. 2017;12 Suppl 2:S255-62, 10.1123/ijspp.20160423. Epub 2016.

8. Gajda R, Biernacka EK, Drygas W. Are heart rate monitors valuable tools for diagnosing arrhythmias in endurance athletes? Scand J Med Sci Sports. 2018;28:496-516, http://dx.doi.org/10.1111/sms.12917. Epub 2017 Jun 16.

9. Gilman MB. The use of heart rate to monitor the intensity of endurance training. Sports Med. 1996;21:73-9.

10. Knoepfli-Lenzin C, Haenggli B, Boutellier U. Optimised heart rate formulae to monitor endurance training in sedentary individuals. J Sports Sci. 2014;32:557-62, http://dx.doi.org/10.1080/02640414.2013.843015.

11. Gellish RL, Goslin BR, Olson RE, McDonald A, Russi GD, Moudgil VK. Longitudinal modeling of the relationship between age and maximal heart rate. Med Sci Sports Exerc. 2007;39:822-9.

12. Tanaka H, Monahan KD, Seals DR. Age-predicted maximal heart rate revisited. J Am Coll Cardiol. 2001;37:153-6. PubMed PMID: 11153730.

13. Suk MH, Yu KH, Shin YA. Comparison of tests for measuring maximal exercise ability in elite swimmers. J Exerc Rehabil. 2016;12:209-15, http://dx.doi.org/10.12965/jer.1632606.303.

14. Kaye DM, Esler MD. Autonomic control of the aging heart. Neuromolecular Med. 2008; 10(3):179-86. doi: 10.1007/s12017008-8034-1. Epub 2008 Mar 13. Review. PubMed PMID: 18338270.

15. Borresen J, Lambert MI. The quantification of training load, the training response and the effect on performance. Sports Med. 2009;39:779-95, http://dx.doi.org/10.2165/11317780-000000000-00000.
16. Bouts AM, Brackman L, Martin E, Subasic AM, Potkanowicz ES. The Accuracy and validity of iOS-based heart rate apps during moderate to high intensity exercise. Int J Exerc Sci. 2018;11:533-40, eCollection 2018.

17. Thomson RL, Bellenger CR, Howe PRC, Karavirta L, Buckley JD. Improved heart rate recovery despite reduced exercise performance following heavy training: A within-subject analysis. J Sci Med Sport. 2016;19:255-9, http://dx.doi.org/10.1016/j.jsams.2015.02.010.

19. Gillinov S, Etiwy M, Wang R, Blackburn G, Phelan D, Gillinov AM, et al. Variable accuracy of wearable heart rate monitors during aerobic exercise. Med Sci Sports Exerc. 2017;49:1697-703, doi:10.1249/MSS. 0000000000001284.

20. McGuigan M. Monitoring training and performance in athletes. Human Kinetics. 2017.

21. Morton RH, Fitz-Clarke JR, Banister EW. Modeling human performance in running. J Appl Physiol. 1990;69:1171-7.

22. Barrett S, Midgley A, Lovell R. PlayerLoad ${ }^{T M}$ : reliability, convergent validity, and influence of unit position during treadmill running. Int J Sports Physiol Perform. 2014;9:945-52, http://dx.doi.org/10.1123/ijspp.2013-0418.

23. lellamo F, Manzi V, Caminiti G, Vitale C, Castagna C, Massaro $M$, et al. Matched dose interval and continuous exercise training induce similar cardiorespiratory and metabolic adaptations in patients with heart failure. Int J Cardiol. 2013;167:2561-5, http://dx.doi.org/10.1016/j.ijcard.2012.06.057.

24. Piepoli MF, Corrà U, Benzer W, Bjarnason-Wehrens B, Dendale P, Gaita D, et al. Cardiac rehabilitation section of the European Association of Cardiovascular Prevention and Rehabilitation Secondary prevention through cardiac rehabilitation: from knowledge to implementation. A position paper from the Cardiac Rehabilitation Section of the European Association of Cardiovascular Prevention and Rehabilitation. Eur J Cardiovasc Prev Rehabil. 2010;17:1-17, http://dx.doi.org/10.1097/HJR.0b013e3283313592.

25. Piña IL, Apstein CS, Balady GJ, Belardinelli R, Chaitman BR, Duscha BD, et al. American Heart Association Committee on exercise, rehabilitation, and prevention. Exercise and heart failure: A statement from the American Heart Association Committee on exercise, rehabilitation, and prevention. Circulation. 2003; 107:1210-25.

26. Wisløff U, Støylen A, Loennechen JP, Bruvold M, Rognmo $\varnothing$, Haram PM, et al. Superior cardiovascular effect of aerobic interval training versus moderate continuous training in heart failure patients: a randomized study. Circulation. 2007;115:3086-94. 